\title{
Dynamical analysis of turbulent liquid phase in two-phase pipe flow
}

\author{
Shahzad Munir ${ }^{\mathrm{a}, *}$, Morgan R. Heikal ${ }^{\mathrm{b}}$, A. Rashid A. Aziz ${ }^{\mathrm{b}}$, Mohana S. Muthuvalu ${ }^{\mathrm{a}}$, Azuraien Jaafar ${ }^{\mathrm{b}}$, \\ Muhammad Israr ${ }^{\mathrm{a}}$ \\ a Department of Fundamental and Applied Science, Universiti Teknologi PETRONAS, Bandar Seri Iskandar, \\ 31750 Tronoh, Perak, Malaysia \\ b Department of Mechanical Engineering, Universiti Teknologi PETRONAS, Bandar Seri Iskandar, \\ 31750 Tronoh, Perak, Malaysia
}

*Corresponding author, e-mail: shahzad_munir@comsats.edu.pk

\begin{abstract}
An experimental study was conducted to evaluate dynamic structures embedded in a liquid and turbulent characteristics of slug flow in a horizontal pipe. Initially, single liquid phase flow was considered at a flow rate of $120 \mathrm{l} / \mathrm{m}$ and later on slug flow was generated by the introduction of a gas phase at the rate of $40 \mathrm{l} / \mathrm{m}$ keeping the liquid phase flow at the same rate. Particle image velocimetry was employed to calculate the two-dimensional instantaneous velocity field of the liquid phase. The turbulent velocity fields and dynamics of the liquid phase flow were investigated by proper orthogonal decomposition analysis. The structures containing the most energy were successfully revealed. The energy distributions of the spatial and temporal modes were also obtained.
\end{abstract}

KEYWORDS: particle image velocimetry, turbulence, proper orthogonal decomposition, spatial modes, temporal modes

\section{INTRODUCTION}

A slug flow pattern is often observed when transporting gas and liquid in horizontal and near horizontal pipelines. It has numerous applications in various industrial situations like oil production fields, in chemical and nuclear reactors. Several intrusive experimental studies have been carried out to understand this important phenomenon and to get knowledge about the dynamical and statistical parameters involved in slug flow ${ }^{1-3}$. Slug flow or any other twophase flow results in turbulence. It involves rapid fluctuations of pressure and velocity in space and time. Along with that it also undergoes changes like heat and momentum transfer, energy production and dissipation. Coherent structures ${ }^{4}$ are considered to be the most prominent characteristic of turbulence that involves vortex bursting and shedding. These structures are responsible for intensifying turbulent mixing phenomenon. A sketch of these coherent structures describing them as horseshoe vortex was given by Theodorsen ${ }^{5}$. Bandyopadhyay ${ }^{6}$ and Head and Bandyopadhyay ${ }^{7}$ experimentally found that those horseshoe vortices are fundamental element of wallbounded turbulent flows. These horseshoe vortices or hairpin-like structures combine into larger-scale structures typically referred to as hairpin vortex packets ${ }^{8,9}$.
The existence of hairpin vortex structures was also supported by Christensen and Adrian ${ }^{10}$, endorsing that these packets effects on the statistical nature of the flow.

Using statistical flow visualization technique, we can identify these coherent structures. Particle image velocimetry (PIV) ${ }^{11}$ is a non-intrusive technique that provides two-dimensional velocity vector field that can be used for identification. This technique also provides an insight into the detailed structure of complex flows. We define a coherent structure by proper orthogonal decomposition (POD). POD is an impartial and statistical technique to define a coherent structure. It is based on Mathematical correlations. Lumley ${ }^{12}$ was the first to investigate turbulent flows using POD analysis. The large-scale behaviour of the flow can be represented by first few modes because they are the only true representative of the flow as they contain information about the most dominant structures. POD modes are the optimal basis of the flow. POD is extensively used in nearly every turbulent phenomenon ${ }^{13-15}$

In this paper, we presented a part from our ongoing project of temporal and spatial characterization of slug flow and the analysis of both liquid and gas phases using decomposition techniques. We considered two cases, one single liquid phase flow at $120 \mathrm{l} / \mathrm{m}$ 


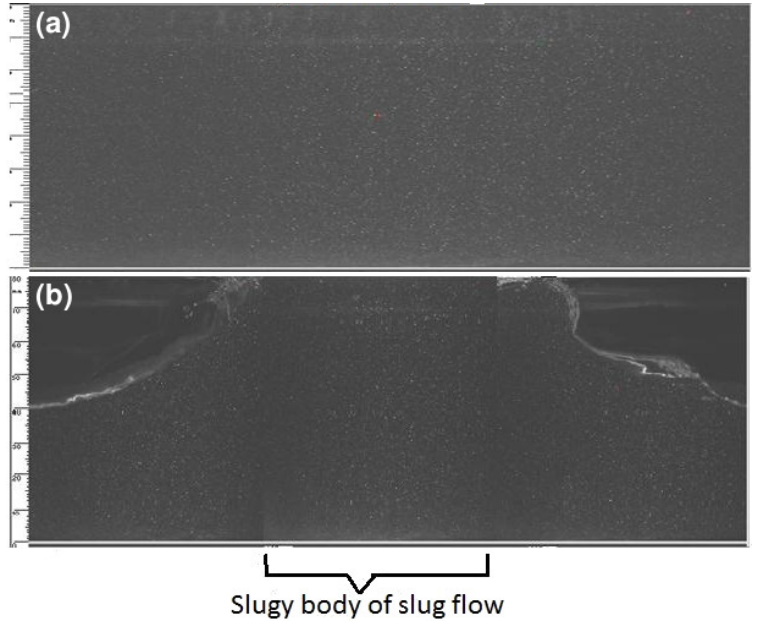

Fig. 1 PIV raw image. (a) Single phase flow, (b) Two phase slug flow.

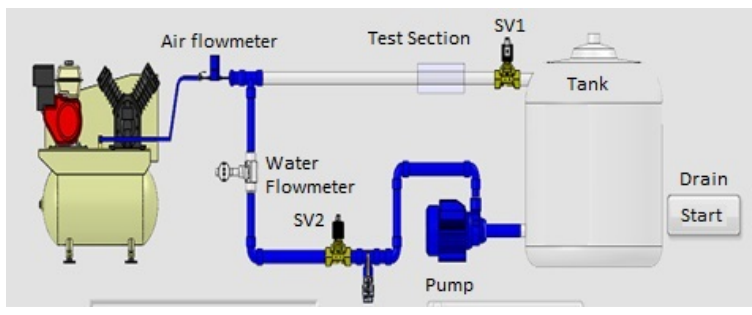

Fig. 2 Experimental facility used for the optical measurements.

and second the liquid slug of slug flow (Fig. 1). The slug flow is generated by introducing gas at $40 \mathrm{l} / \mathrm{m}$ while keeping liquid flow rate at $120 \mathrm{l} / \mathrm{m}$. A total of 205 snapshots were collected for single phase liquid flow and slug flow. For later, 43 snapshots of slug body were gathered out of 205 . The images of this liquid slug almost look like single phase liquid flow but they are not the same as the former is influenced by gas phase. That is why we chose these cases for analysis and comparison. PIV provides measurements of the two-dimensional velocity fields of the liquid phase for both cases. POD analysis technique was applied to the numerical data of instantaneous velocity of both cases. We aimed to extract the information about large structures and their overall effect on the dynamics of the flow. This technique also provides details of the energy contained in these structures.

\section{EXPERIMENTAL SETUP AND METHOD}

The experimental set-up is shown in Fig. 2. The length of the transparent Plexiglas pipe is $6 \mathrm{~m}$ and has an internal diameter $74 \mathrm{~mm}$. The gas-phase enters horizontally along the transparent pipe through a $T$ shaped junction designed to be mixing phase. The liquid and gas phase is represented by water and air, respectively. To circulate water into the closed flow loop, a centrifugal pump is used. The maximum flow rate of pump is $500 \mathrm{1} / \mathrm{m}$. A water storage tank is also installed having capacity of $800 \mathrm{l}$. The air is injected through a nozzle of $2 \mathrm{~mm}$ inner diameter and for that purpose an air compressor of 8 bars maximum pressure is used. The liquid flow rate is measured by turbine mass flow meter. A USB acquisition control system is attached with the flow loop to perform all operations with a computer. The test section is a rectangular box made of the same transparent Plexiglas pipe. This test section is placed at $3.5 \mathrm{~m}$ from the inlet for optical measurements. Experiment for single phase liquid flow at $120 \mathrm{l} / \mathrm{m}$ is performed initially. After that slug flow is generated by injecting compressed air at $40 \mathrm{l} / \mathrm{m}$. Instantaneous measurements are performed by combining particle image velocimetry (PIV) and laser induced fluorescence. Fluorescent tracer particles coated with Rhodamine $\mathrm{B}$ are used in liquid phase. They have a size 1-20 $\mu \mathrm{m}$, density $1.19 \mathrm{~g} / \mathrm{cm}^{3}$ and a refractive index of about 1.479. A double pulsed Nd:YAG is used to illuminate these particles with a green laser sheet of wavelength $532 \mathrm{~nm}$. These particles excite and scatter the light of wavelength greater than $570 \mathrm{~nm}$. A CCD camera is used to capture the movement of the tracer particles. The camera has a low pass optical filter with cut off wavelength $570 \mathrm{~nm}$ to detect the orange light emitted from the tracer particles. Some of 205 double images frames are collected and stored. For the analysis of these images, we used Dantec Dynamics Flow manager. The interested region is masked out and cross-correlated using 50\% overlap. The interrogation area (IA) is $64 \times 64$ pixels. Moving average filter is used to validate the spurious vectors. The average filter is of size $3 \times 3$ IA. A velocity vector map of $39 \times 31$ vectors representing the instantaneous velocity fields is obtained for both cases.

\section{Proper orthogonal decomposition}

POD is best known for the identification of dominant structures, "coherent structures" in flows. With a few number of modes, POD reconstructs low-dimensional models that best represents main flow dynamics. In POD, the deviations from mean can be decomposed and written in a linear sum of orthogonal eigenfunctions. These eigenfunctions represents coherent structures in the field. The two-point spatial correlation is determined by PIV data. Suppose we have a total number of $M$ snapshots of 2-D velocity fields at 


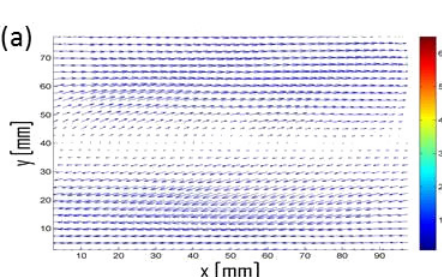

(c)

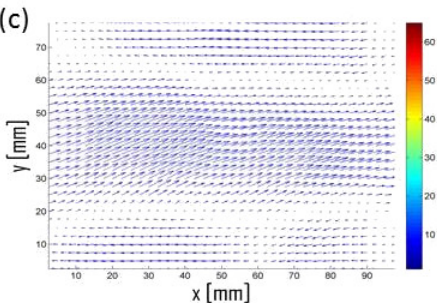

(e)

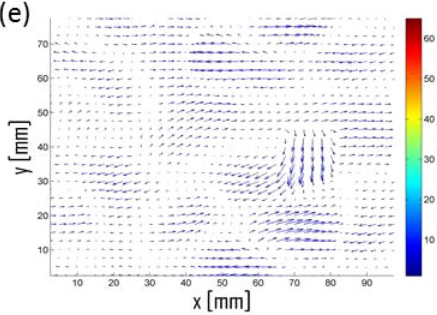

(g)

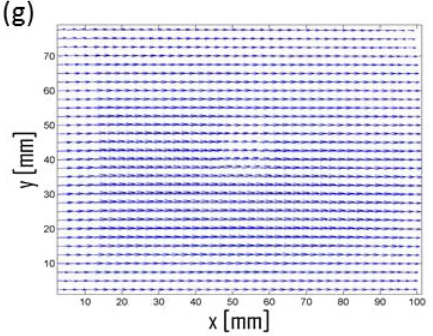

(b)

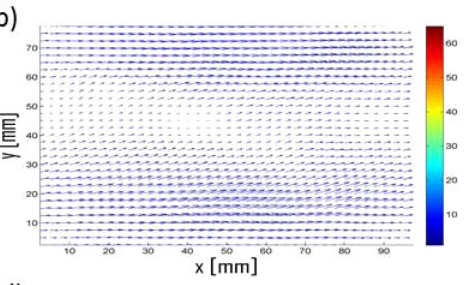

(d)

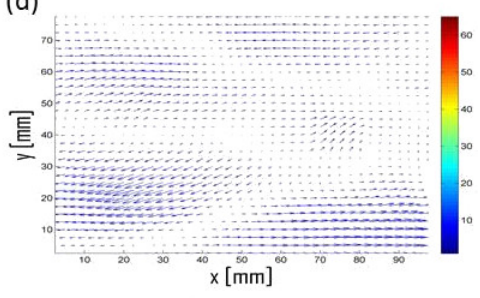

(f)

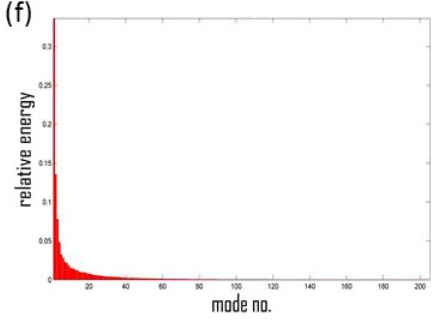

(h)

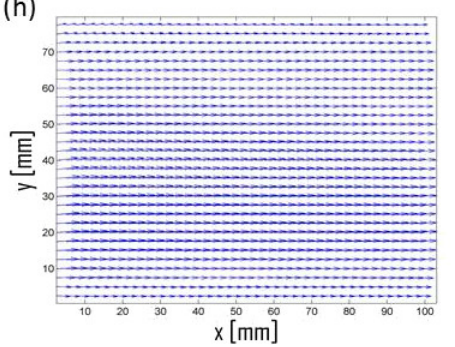

Fig. 3 Case I. (a-d) POD modes 1-4. (e) POD mode 20. (f) Energy spectra. (g) Reconstructed field. (h) Snapshot.

times $t_{1}, \ldots, t_{M}$. The fluctuations $u^{\prime}(x, t)$ from timeaveraged part $U(x)$, for 2-D velocity fields $u(x, t)$, can be calculated from,

$$
u(x, t)=U(x)+u^{\prime}(x, t) .
$$

In POD method of snapshot, we try to get a basis $\left\{\varphi_{k}(x)\right\}$ in which we can optimally describe the ensemble $\left\{u_{n}(x)\right\}$. The solution of the following integral equation gives the orthogonal spatial modes,

$$
\int_{0}^{1}\left\langle u(x) u\left(x^{\prime}\right)^{T}\right\rangle \varphi\left(x^{\prime}\right) \mathrm{d} x^{\prime}=\gamma \varphi(x),
$$

where

$$
u(x) u\left(x^{\prime}\right)^{T}=R\left(x, x^{\prime}\right)=\sum \gamma_{j} \varphi_{j}(x) \varphi\left(x^{\prime}\right)^{T} .
$$

Here $R\left(x, x^{\prime}\right)$ is a two point spatial correlation function $M \times M$ matrix, $\varphi_{j}$ is an eigenfunction representing the dominant turbulent structure and $\gamma_{j}$ is the eigenvalue. In this snapshot POD, these eigenvalues represents the fluctuating energy associated with large-scale structures. The total fluctuation energy can be found out by the sum of the eigenvalues. Flow reconstruction of the original is represented by few number of modes,

$$
u(x)=\sum_{n} \gamma_{n} \varphi_{n}(x) .
$$

\section{RESULTS}

Fig. 3(a-e) shows the POD modes for single phase liquid flow. It can be seen very easily that the POD has successfully extracts the large structures having maximum energy. For the first few modes the curve falls sharply, but become less and less steep the higher the mode number (Fig. 3f). The first, most energetic modes represent the large dominant structures of the flow. The lower energy levels indicating smaller flow 
(a)

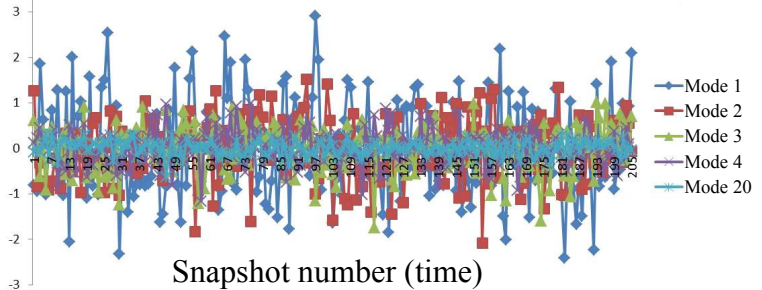

(b)

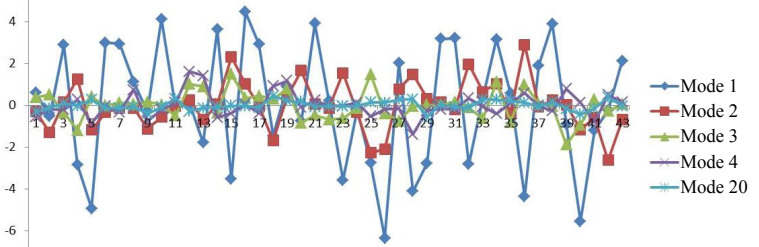

Snapshot number (time)

Fig. 4 Time variation of POD coefficients. (a) Case I. (b) Case II.

structures give us more detailed information about the flow.

Modes 1 and 2 which together have $47.7 \%$ of the total energy consist of large dominant structures. Mode 1 reveals the movement of low momentum fluid away from the wall and high momentum fluid towards the upper wall. In the centre of the field of view we can also see the zero velocity region. Mode 2 identifies only the large-scale movement of high momentum fluid towards the wall. This mechanism of the movement of high and low momentum fluid occurs frequently in the following modes. Mode 3 has $7.7 \%$ and mode 4 has $4.8 \%$ of the energy. Mode 20 has almost $0.7 \%$ energy which represents information about the presence of vortices. If we keep on going with high mode numbers we will get quite low energy but high details of vortices embedded in it. The lower the energy level, the more detail is offered in a given POD mode. As we go towards higher mode number like 100 or 200 , these modes contain information about small-scale motion only and as such cannot be interpreted as representative of flow structure. Fig. $3 \mathrm{~g}$ represents the reconstructed velocity field. Because POD depends on fluctuating velocity, the velocity field which will construct from this will only represents fluctuation. So in order to get a complete velocity field we have to add mean velocity with this fluctuation. In this case we reconstructed the velocity by using ten modes that had the combined energy of $73.6 \%$ and it matches well with snapshot Fig. $3 \mathrm{~h}$. The temporal modes or POD coefficients of spatial modes for case I are shown in Fig. 4a. The first temporal mode is oscillating with much smaller frequency that is related to spatial POD mode 1 which contains maximum energy. As we move on further we can see that temporal mode 20 oscillates with higher frequency and smaller amplitudes indicating the lowenergy level and small-scale flow structures.

In case II, the slug flow was generated by introducing the compressed air at $40 \mathrm{1} / \mathrm{m}$. Proper orthogonal decomposition was used to investigate the spatial and temporal structures of turbulent water flow. Fig. 5(a-d) shows the POD eigenmodes and energy graph for the case II. The spatial modes achieved were quite different compared with those shown in Fig. 3(ae) of case I. The first spatial mode Fig. 5a represents high momentum fluid moving from left to right and towards the wall. We conjecture that it might be due to the size of the structures that is bigger than the field of view. The second spatial mode contains different dynamics (Fig. 5b). Three counter vortices appeared in third spatial mode (Fig. 5c). Many small scale vortices can be seen in spatial mode 20 (Fig. 5d). From energy point of view, first two spatial modes contained $75 \%$ of the total energy and just $0.4 \%$ energy retained in mode 20 (Fig. 5e). Reconstructed velocity field was obtained by using just first two high energy containing modes (Fig. 5f). As far as the temporal modes are concerned, the first temporal mode is oscillating with the maximum amplitude highlighting the dominance of spatial mode 1 (Fig. 4a). However, temporal mode 20 had small amplitude but rapid fluctuations (Fig. 4b).

\section{CONCLUSIONS}

The temporal and spatial characterization of turbulent liquid phase in both single phase flow and two-phase slug flow were studied. In this regard, an experimental study was conducted using particle image velocimetry and proper orthogonal decomposition techniques. Slug flow was generated by the introduction of air at $40 \mathrm{l} / \mathrm{m}$. PIV gave us the two-dimensional instantaneous velocity fields for liquid phase in both cases. Proper orthogonal decomposition analysis offers new look into the turbulent flow structures embedded in the liquid phase. It successfully extracts information about large scale dominant structures by determining the high energy modes. Due to the limited amount of energy contained in the small-scale motions, these are not captured until very high mode numbers. In case I, the first two modes have $47.7 \%$ of the total energy while in case II they have 75\% energy. Modes 1 and 2 for case I has identified the fluctuating structure in the streamwise direction, however for case II mode 1 mainly highlights the large momentum flow. The first temporal modes for both cases oscillates with high 
(a)

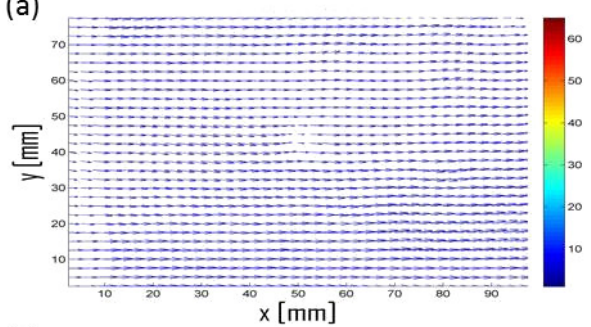

(c)

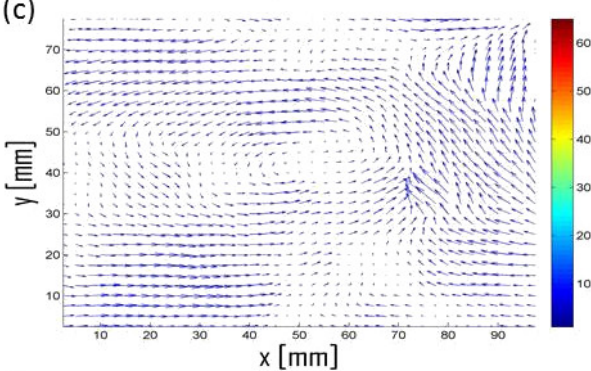

(e)

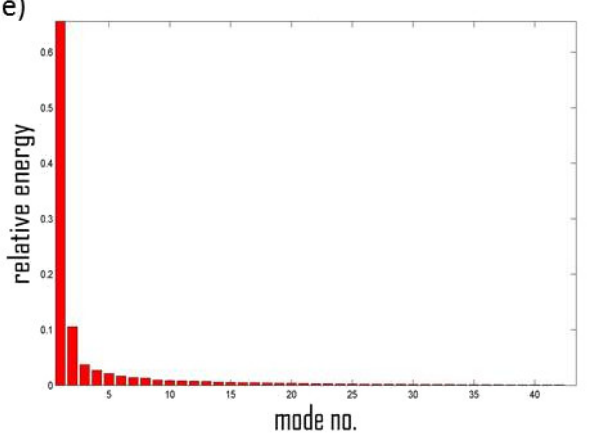

(b)

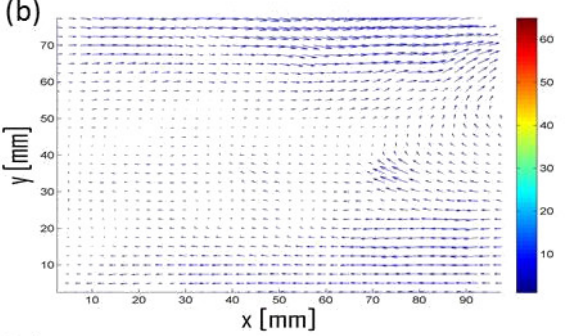

(d)

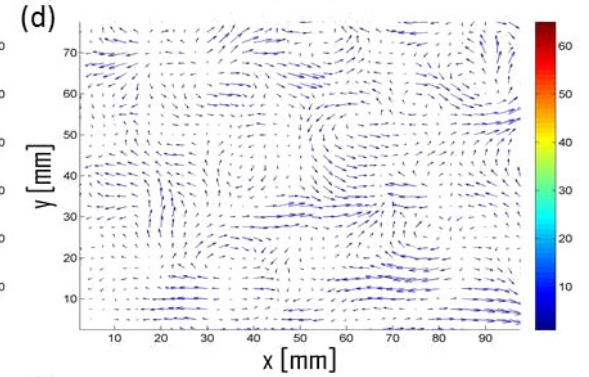

(f)

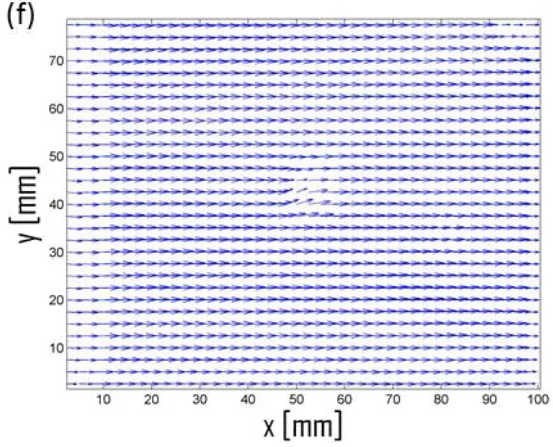

Fig. 5 Case II. (a-c) POD modes 1-3. (d) POD mode 20. (e) Energy spectra. (f) Reconstructed velocity field.

amplitude which decreases for higher order modes. Since the energy was distributed among a large number of eigenmodes we opt to utilize first ten modes for the reconstruction of the flow field for case I, whereas for case II only the first two dominant and energetic modes are used for the reconstruction and for both cases the reconstruction matched well with the original velocity field.

Acknowledgements: This work was supported by YUTP (015-3AA-A61) and URIF (35/2011) internal grants from Universiti Teknologi PETRONAS (UTP). The first and last author are supported by Graduate Assistantship scheme UTP.

\section{REFERENCES}

1. Tay BL, Thorpe RB (2004) Effects of liquid physical properties on the forces acting on a pipe bend in gasliquid slug flow. Chem Eng Res Des 82, 344-56.

2. Kadri U, Henkes RAWM, Mudde RF, Oliemans RVA (2011) Effect of gas pulsation on long slugs in hori- zontal gas-liquid pipe flow. Int $J$ Multiphas Flow 37, 1120-8.

3. Hubbard M (1965) An analysis of horizontal gas-liquid slug flow. PhD thesis, University of Houston, Texas, USA.

4. Cantwell BJ (1981) Organized motion in turbulent flow. Annu Rev Fluid Mech 13, 457-515.

5. Theodorsen $\mathrm{T}$ (1952) Mechanism of turbulence. In: Proceedings of the Second Midwestern Conference on Fluid Mechanics, Ohio State Univ, Columbus, pp 1-18.

6. Bandyopadhyay P (1980) Large structure with a characteristic upstream interface in turbulent boundary layers. Phys Fluids 23, 2326-7.

7. Head MR, Bandyopadhyay P (1981) New aspects of turbulent boundary-layer structure. J Fluid Mech 107, 297-338.

8. Zhou J, Adrian RJ, Balachandar S, Kendall TM (1999) Mechanisms for generating coherent packets of hairpin vortices in channel flow. J Fluid Mech 387, 353-96.

9. Adrian RJ, Christensen KT, Liu ZC (2000) Analysis and interpretation of instantaneous turbulent velocity fields. Exp Fluid 29, 275-90.

10. Adrian RJ, Christensen KT (2001) Statistical evidence 
of hairpin vortex packets in wall turbulence. $J$ Fluid Mech 431, 433-43.

11. Adrian RJ, Westerwheel J (2011) Particle Image Velocimetry, Cambridge Univ Press.

12. Lumley JL (1967) The structure of inhomogeneous turbulent flows. In: Yaglom AM, Tatarski VI (eds) Atmospheric Turbulence and Radio Wave Propagation, Nauka, Moscow, pp 166-78.

13. Berkooz G, Holmes P, Lumley JL (1993) The proper orthogonal decomposition in the analysis of turbulent flows. Annu Rev Fluid Mech 25, 539-75.

14. Hellstrom LHO, Sinha A, Smits AJ (2011) Visualizing the very-large-scale motions in turbulent pipe flow. Phys Fluids 23, ID 011703.

15. Meyer KE, Pedersen JM, Ozcan O, Meyer KE, Pedersen JM, Ozcan O (2007) A turbulent jet in crossflow analysed with proper orthogonal decomposition. J Fluid Mech 583, 199-227. 